\title{
A soft robotic supernumerary finger and a wearable cutaneous finger interface to compensate the missing grasping capabilities in chronic stroke patients
}

\author{
Irfan Hussain $^{1}$, Leonardo Meli ${ }^{1}$, Claudio Pacchierotti ${ }^{2}$ and Domenico Prattichizzo ${ }^{1,3}$
}

\begin{abstract}
Stroke survivors who experience severe hemiparesis often cannot completely recover the use of their hand and arm. Many of the rehabilitation devices currently available are designed to increase the functional recovery right after the stroke when, in some cases, biological restoring and plastic reorganization of the central nervous system can take place. However, this is not always the case. Even after extensive therapeutic interventions, the probability of regaining functional use of the impaired hand is low. In this respect, we present a novel robotic system composed of a supernumerary robotic finger and a wearable cutaneous finger interface. The supernumerary finger is used to help grasping objects while the wearable interface provides information about the forces exerted by the robotic finger on the object being held. We carried out two experiments, enrolling 16 healthy subjects and 2 chronic stroke patients. Results showed that using the supernumerary finger greatly improved the grasping capabilities of the subjects. Moreover, providing cutaneous feedback significantly improved the performance of the considered task and was preferred by all subjects.
\end{abstract}

\section{INTRODUCTION}

Long-term disabilities of the upper limb affects millions of stroke survivors [1]. More than $80 \%$ of individuals who experience severe hemiparesis after stroke cannot completely recover hand and arm use [2]. The improvement of the paretic hand functionality plays a key role in the functional recovery of stroke patients with a paretic upper limb [3]. Different motor impairments can affect the hand both at motor execution and motor planning/learning level, including weakness of wrist/finger extensors, increased wrist/finger flexors tone and spasticity, co-contraction, impaired finger independence, poor coordination between grip and load forces, inefficient scaling of grip force and peak aperture, and delayed preparation, initiation, and termination of object grip [4]. In the last two decades, several rehabilitation teams have started integrating robotic-aided therapies in their rehabilitation projects. Such treatments represent a novel and promising approach in rehabilitation of the post-stroke paretic upper limb. The use of robotic devices in rehabilitation can provide high-intensity, repetitive, task-specific and interactive treatment of the impaired upper limb, and can serve as an objective and reliable means of monitoring patient

This research has received funding from the European Union Seventh Framework Programme FP7/2007-2013 under grant agreement n601165 of the project "WEARHAP" and from the European Union's Horizon 2020 Research and Innovation Programme under Grant Agreement n ${ }^{\circ} 688857$ of the project "SoftPro".

${ }^{1}$ Dept. Information Eng. and Mathematics, Univ. Siena, Siena, Italy.

${ }^{2}$ CNRS at Irisa and Inria Rennes Bretagne Atlantique, Rennes, France.

${ }^{3}$ Dept. Advanced Robotics, Istituto Italiano di Tecnologia, Genoa, Italy.

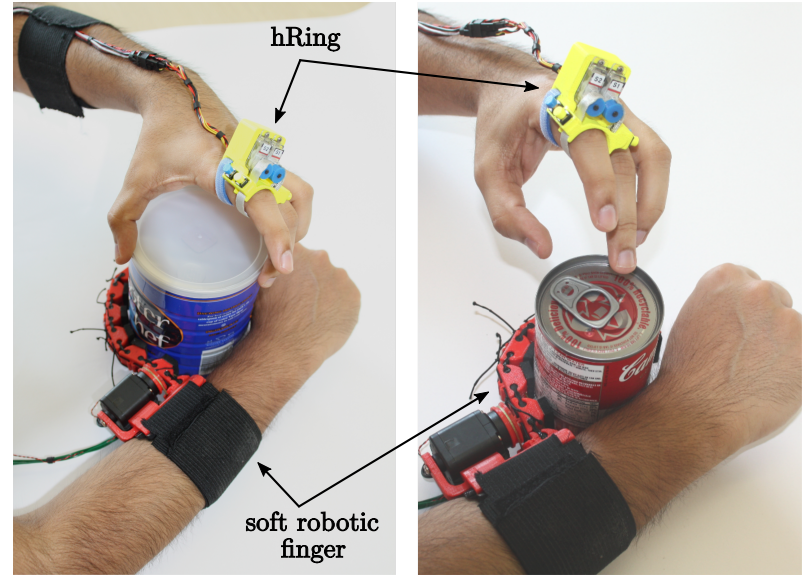

(a) Opening a jar of coffee (left) and tomato (right).

Fig. 1. The system is composed of a supernumerary robotic finger and a wearable cutaneous finger interface, called hRing. The picture shows the integrated system used by a patient in Activities of Daily Living (ADL). The hRing is used to control the opening/closing motion of the robotic finger and to provide the wearer with information about the forces exerted by the robotic finger.

progress [5], [6]. Most of the proposed devices for hand and arm rehabilitation are designed to increase functional recovery in the first period after the stroke when, in some cases, biological restoring and plastic reorganization of the central nervous system may take place [7]. However, even after extensive therapeutic interventions in acute rehabilitation, the probability of regaining functional use of the impaired hand is low [8].

For this reason, our group has recently started to study innovative robotic devices for the compensation of hand functions in chronic stroke patients [9], [10], [11], [12]. We devised wearable robotic supernumerary fingers that can be used as an active compensatory tool for grasping objects. These devices can be easily worn on the paretic forearm by means of an elastic band, so that the robotic finger and the paretic hand can act like the two parts of a gripper working together to hold an object. To further improve their wearability, these fingers can be also shaped into a bracelet when not used. The user can then control the flexion/extension of the robotic finger through different means, such as an EMG interface placed on the patient forehead [13] or a hardware switch [10], [14], [15].

These above mentioned solutions have shown promising results, re-enabling impaired users to grasp objects they would have not been able to grasp otherwise. Despite this, 
they do not provide any information about the forces exerted by the supernumerary limb on the environment, which is known to be useful [16], [17], [18]. Haptic feedback is particularly important for post-stroke patients suffering from tactile anesthesia, or anaphia [19], in the hand contralateral to the stroke, which is the one that should provide force information about the supernumerary limb. For this reason, there have been attempts to restore the sense of touch in patients affected by this deficit. A very first result has been presented in [10], [14], where the authors developed a vibrotactile ring to provide information about the status of a robotic supernumerary finger.

Although promising, the design of the supernumerary fingers presented in [13], [11], [9], [20] only considered fully-actuated supernumerary devices, which used as many motors as joints, resulting in quite complex and cumbersome systems. Moreover, the haptic interfaces for supernumerary limbs presented in [10], [14] considered only very simple vibrotactile interfaces, which prevented them from conveying any rich force information.

For this reason, in this paper, we present an innovative soft supernumerary underactuated robotic finger coupled with a wearable skin stretch cutaneous device, shown in Figs. 1 and 2. The supernumerary finger is controlled by only one motor, and its soft structure enables it to adapt to the object being grasped, resulting in a gentle but stable grasp. The haptic interface is able to provide normal, skin stretch, and vibrotactile stimuli at the wearer's finger, relying rich information about the forces exerted by the supernumerary finger on the environment. A live demonstration of this system during the 2016 IEEE Haptics Symposium in Philadelphia, PA, USA, received the "Best Demonstration Award".

\section{The Supernumerary Robotic FInger (SRF)}

The supernumerary robotic finger is a wearable robotic extra finger, that can be used by chronic stroke patients to compensate for the missing functions of their paretic hand [21]. It is composed of an underactuated modular structure to be worn on the paretic forearm by means of an elastic band.

The final prototype used in this work is the result of a long interaction between our group of engineers, the clinicians of the Azienda Ospedaliera Universitaria Senese, and a small group of keen chronic stroke patients.

1) Description: The device consists of two main parts: a modular flexible finger and a support base, as shown in Fig 2b. The support base is realized in ABS (Acrylonitrile butadiene styrene) using rapid prototyping techniques. It houses one servo motor and an elastic band used to wear the device on the forearm. Rubber spacers are used to increase the comfort and grip of the device on the forearm. The flexible finger is composed of seven identical modules. Each module consists of an ABS part that acts as a rigid link and a 3D printed TPU (thermoplastic polyurethane) part that acts as a flexible joint. Finally, a soft rubber layer increases the friction at the contact area. The modules are connected to each other by sliding the thermoplastic polyurethane inside

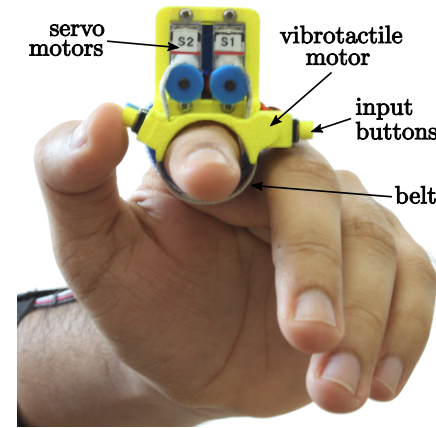

(a) hRing haptic interface.

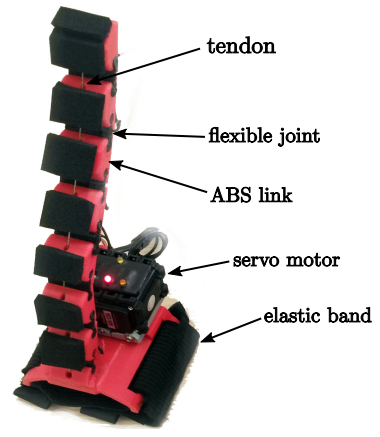

(b) Supernumerary robotic finger.
Fig. 2. The hRing and the supernumerary robotic finger.

the ABS, making the assembling process very easy. $2 \mathrm{~mm}$ holes in the rigid ABS links allows the passage of a tendon that connects all the modules to the servo motor fixed to the support base. The servomotor, a Dynamixel servo MX-28T, drives the flexion/extension of the finger by pulling/releasing the tendon.

The robotic finger has been also designed thinking about its wearability. In fact, it can be easily worn by means of an elastic band, as shown in Fig. 1. It can be also wrapped up on the arm as a bracelet when not used. The switching between working and resting positions is achieved through a passive rotatable locking mechanism.

2) Compliance: Due to the flexible modules, the robotic finger is compliant, making it capable to easily adapt to different shapes during the grasp [22].

During grasping, the mechanical compliance of the soft modules leads to an adaptation of the finger's configuration to the object's shape. Shape adaptation is known to increase grasp performance by compensating for the uncertainties in sensing and actuation, helping to achieve a stable grasp [23].

In our previous works, we considered a fully-actuated fully-sensorized robotic device with rigid links [13], [11]. The closing trajectory of the finger was controlled by the motion of each independent actuator. Advanced control techniques, based on the finger sensory information, were needed to achieve a suitable grasping trajectory for shape adaptation during grasping [14]. With the proposed soft finger, thanks to its flexible design, it is easier to adapt to the grasped object, ensuring a more stable grasp. Moreover, thanks to its underactuated structure, we were able to achieve a more compact and robust design, as well as a simpler control action. Table I shows the technical details of the proposed supernumerary robotic finger.

\section{THE HRING WEARABLE INTERFACE}

To provide the patient with information about the forces exerted by the supernumerary robotic finger to the environment, we used a cutaneous skin stretch device for the proximal finger phalanx. Wearability, comfort, ease of use, and effectiveness were the main requirements for the design of this haptic device [24]. 
TABLE I

TECHNICAL DETAILS OF THE SUPERNUMERARY ROBOTIC FINGER

\begin{tabular}{lr}
\hline Dimensions & \\
\hline Module & $20 \times 31 \times 12 \mathrm{~mm}^{3}$ \\
\hline Total length of finger (on arm) & $180 \mathrm{~mm}$ \\
\hline Diameter smallest graspable obj. & $180 \mathrm{gm}$ \\
\hline Weights & $2.5 \mathrm{Nm} \mathrm{@} 12 \mathrm{~V}$ \\
\hline Total (finger + support base) & $8 \mathrm{~mm}$ \\
\hline Actuator & $1.4 \mathrm{~A} @ 12 \mathrm{~V}$ \\
\hline Max. torque & $300 \mathrm{deg}$, endless turn \\
\hline Pulley radius & $684 \mathrm{deg} / \mathrm{sec}$ \\
\hline Max. current & $40 \mathrm{~N}$ \\
\hline Max. operating angles & $2.4 \mathrm{~kg}$ \\
\hline Max. non-loaded velocity & $13 \mathrm{~N} @$ dia=65 mm \\
\hline Max. Force at fingertip & \\
\hline Max. payload & \\
\hline Max. horizontal resistive force & \\
\hline
\end{tabular}

1) Description: The hRing is shown in Fig. 2a. It is composed of a static part, that houses two servo motors and two pulleys, and a fabric belt, that applies the requested stimuli to the finger. A strap band is used to secure the device on the finger proximal phalanx. We used two PWMcontrolled HS-40 servomotors (HiTech, Republic of Korea). The device weights $38 \mathrm{~g}$ for $30 \times 43 \times 25 \mathrm{~mm}$ dimensions.

2) Haptic feedback: The working principle of the device is depicted in Fig. 3. Similarly to the principle proposed by Minamizawa et al. [25], when the two motors rotate in opposite directions, the belt is pulled up, providing a force normal to the finger (left side of Fig. 3). On the other hand, when motors spin in the same direction, the belt applies a shear force to the finger (right side of Fig. 3). Of course, these two movements can be combined together to provide at the same time shear and normal stimuli.

However, although the hRing can apply both normal and shear forces, in this paper, we used it to only apply forces normal to the finger skin, similarly to [26]. This is mainly due to the fact that we have not added any multi-DoF force sensor on the robotic finger, with the objective of improving its wearability and portability. As detailed below, we estimate the contact force solely from the load of the finger's motor. Nonetheless, as also mentioned in Sec. VI, in the future, we plan to add an ATI 6-DoF force/torque sensor on the distal module of the supernumerary finger, to be able to accurately measure the forces exerted by the finger on the environment.

The relationship between the belt displacement and the force applied to the human finger proximal phalanx, $f_{h}$, is

$$
\Delta d=\frac{f_{h}}{K}
$$

where $\Delta d \in \mathbb{R}$ is the displacement of the belt toward the skin since it first made contact with the fingertip, and $K \in \mathbb{R}$ is the finger phalanx stiffness value [18], [27].

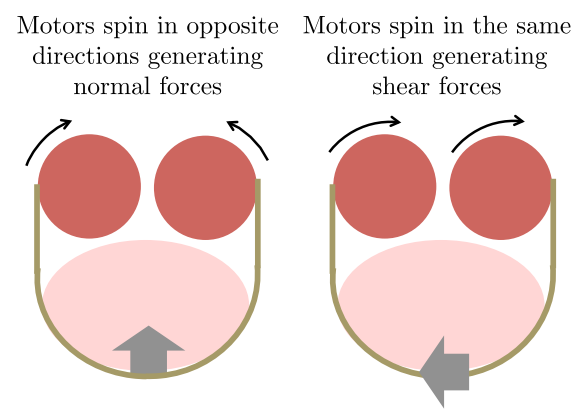

Fig. 3. Device actuation principle. When the motors spin in opposite directions, the belt presses into the user's finger (left), while when the motors spin in the same direction, the belt applies a shear force to the skin (right)

The force exerted by the supernumerary finger on the environment is evaluated by considering the load of the motor $\tau_{m}$,

$$
f_{h}=\frac{f_{h, M}}{\tau_{m, M}} \tau_{m}
$$

where $\tau_{m} \in\left[0, \tau_{m, M}\right] \mathrm{Nm}, \tau_{m, M}=2.5 \mathrm{Nm}$ is the maximum torque the motor can provide (see Tab. I), and $f_{h, M}=9.4 \mathrm{~N}$ is the maximum force the hRing can apply on the finger skin. $f_{h, M}$ was measured positioning an ATI Nano force/torque sensor between the belt and the rigid structure of the hring and applying the maximum opposite rotation of both servo motors, i.e., the maximum squeezing force.

\section{CONTROL}

Pressing the blue (proximal) button on the external side of the hRing (see Figs. 1 and 2) initiates the flexing procedure of the robotic finger. The finger will then close until a contact with an object is detected. As soon as the contact happens, the robotic finger stops its flexion and the hRing generates a short vibration burst to notify the subject. If the subject presses the blue button again, the finger increases the grasping force on the object. During this process, the hRing belt squeezes the user's finger proportionally to the grasp force applied by the robotic finger on the environment (see Sec. III). If the grasping force exerted by the robotic finger reaches the maximum force applicable by the motor, a double vibration burst alerts the user. When the patient is satisfied with the grasping configuration, he or she can proceed with the task. Finally, pressing the yellow (distal) button on the hRing will initiate the opening procedure of the robotic finger. A video of the integrated system is available as supplemental material.

\section{EXPERIMENTS}

In order to demonstrate the effectiveness of our system, we carried out two experiments, enrolling sixteen healthy subjects and two post-stroke patients.

\section{A. Experiment \#1: pick and place with healthy subjects}

1) Subjects: Sixteen healthy participants (12 males, 4 females, age range 21 - 32) took part to the experiment, all of whom were right-handed. Nine of them had previous experience with haptic interfaces. 
TABLE II

DETAILS ON THE OBJECTS USED FOR THE EXPERIMENT

\begin{tabular}{lcc}
\hline Object & Weight $(\mathrm{g})$ & Dimensions $(\mathrm{cm})$ \\
\hline Dice & 10 & $7 \times 7 \times 7$ \\
\hline Glass & 3 & $7 \times 7 \times 8$ \\
\hline Cube \#1 & 8 & $2.5 \times 2.5 \times 2.5$ \\
\hline Cube \#2 & 8 & $2.5 \times 2.5 \times 2.5$ \\
\hline Sauce can & 348 & $6.5 \times 6.5 \times 10$ \\
\hline Toy \#1 & 160 & $7.5 \times 7.5 \times 10$ \\
\hline Toy \#2 & 107 & $6 \times 6 \times 9.5$ \\
\hline
\end{tabular}

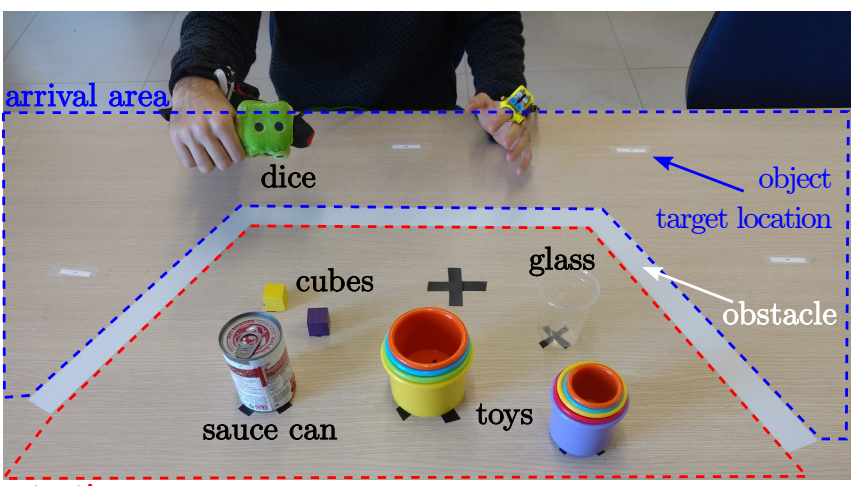

starting area

Fig. 4. Experiment \#1: pick and place. Subjects were asked to pick, lift, and place all the objects correctly, being as fast as possible and using only their right (impaired) arm. The hRing was used to open/close the supernumerary finger and to receive haptic feedback.

2) Setup: The experimental setup is shown in Fig. 4. It is composed of our supernumerary finger, worn on the right forearm, and the hRing cutaneous interface, worn on the proximal phalanx of the left index finger. As for this experiment we enrolled only healthy subjects, we asked them to mimic the post-stroke hemiparesis of their right hand by keeping the hand closed in a fist. Subjects were then asked to stay in front of a table where 5 different objects were placed. Details on the objects are reported in Table II. The target locations for the objects were marked on the table. Subjects had to pick, lift, and place all the objects correctly, being as fast as possible and using only their right (impaired) arm. Subjects could move the objects in any order they prefer. To prevent subjects from dragging the objects, we placed an obstacle between the starting and target objects' locations. A video of the experiment is available as supplemental material.

3) Methods: We carried out the pick and place task in three different experimental conditions: no grasping compensation $(\mathrm{N})$, grasping compensation using the supernumerary finger (S), grasping compensation using the supernumerary finger and the hRing haptic interface (SH). Subjects performed the experiment two times per condition, ending up with 6 randomized repetitions of task per subjects.

When no grasping compensation $(\mathrm{N})$ was considered, subjects did not wear the supernumerary finger and the hRing interface.
TABLE III

EXPERIMENT \#1: SUCCESS RATE.

\begin{tabular}{lcc}
\hline Condition & Success rate & $\begin{array}{c}\text { Objects placed at the time limit } \\
\text { (mean } \pm \text { standard dev.) }\end{array}$ \\
\hline $\mathrm{N}$ & $0 \%$ & $\begin{array}{c}3.67 \pm 0.49 \\
\text { (no one picked up the small cubes) }\end{array}$ \\
\hline $\mathrm{S}$ & $100 \%$ & 5 \\
\hline $\mathrm{SH}$ & $100 \%$ & 5 \\
\hline
\end{tabular}

When grasping compensation using the supernumerary finger ( $\mathrm{S}$ ) was considered, subjects were asked to wear the supernumerary finger on their right forearm and the hRing on the proximal phalanx of their left index fingers. The hRing was only used to control the opening/closing motion of the supernumerary finger, as described in Sec. IV, and provided no haptic feedback.

When grasping compensation using the supernumerary finger and the hRing haptic interface ( $\mathrm{SH}$ ) was considered, subjects were asked again to wear the supernumerary finger on their right forearm and the hRing on the proximal phalanx of their left index fingers. This time the hRing was used to both control the opening/closing motion of the supernumerary finger, as described in Sec. IV, and to provide haptic feedback about the forces exerted by the supernumerary finger to the environment and about the status of the grasping procedure, as described in Secs. III and IV.

In all these conditions, subjects were asked to keep their right hand closed in a fist to mimic the hemiparesis of the hand.

4) Results: As a measure of performance, we evaluated (1) the success rate of the pick and place task, (2) the completion time, (3) the force applied by the supernumerary finger to the object being grasped, and (4) the perceived effectiveness of the three conditions.

Table III shows the success rate for the considered task. The task was considered successful if all the objects were correctly placed within a time limit of 3 minutes. No one was able to pick up the two small cubes without the supernumerary finger (condition $\mathrm{N}$ ). Due to this result, for the next two metrics, we only considered conditions using the supernumerary finger ( $\mathrm{S}$ and $\mathrm{SH}$ ).

Fig. 5a shows the completion time. The collected data passed the Shapiro-Wilk normality test. We ran a pairedsamples t-test and the condition $\mathrm{S}$ elicited a statistically significant increase in completion time compared to condition SH, $t(15)=2.153, p=0.048$.

Fig. $5 \mathrm{~b}$ shows the mean load exerted by the supernumerary finger on the environment, registered by the servo motors, considering only instants when the load was more than zero. Fig. 5c shows the same mean load split object by object (objects are listed in Tab. II). We ran a two-way repeatedmeasures ANOVA. Feedback conditions (S vs. SH) and objects were treated as within-subject factors. The data passed the Shapiro-Wilk normality test. Mauchly's Test of sphericity indicated that the assumption of sphericity had not been violated for the objects factor $\left(\chi^{2}(9)=10.066, p=0.349\right)$. 


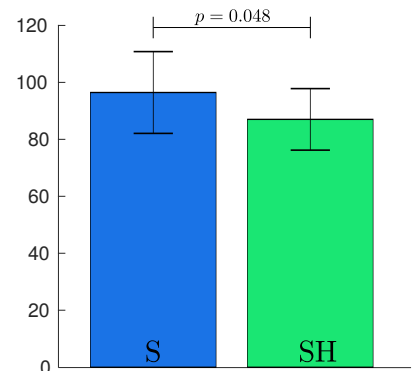

(a) Completion time (s).

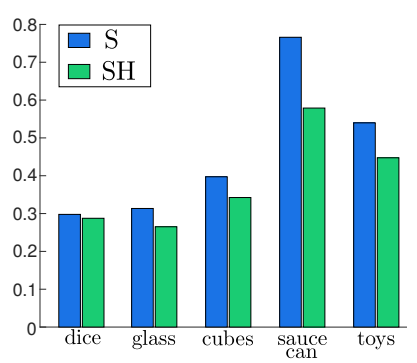

(c) Motor load per object $(\mathrm{Nm})$.

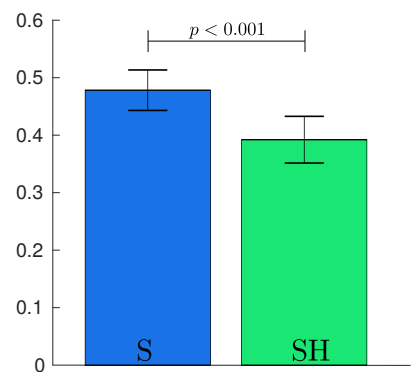

(b) Motor load (Nm).

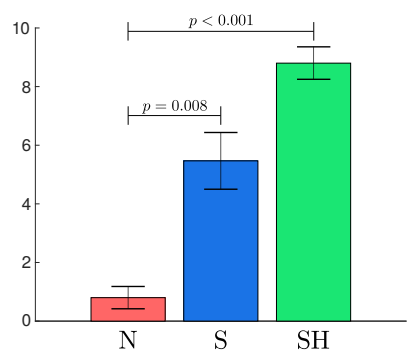

(d) Perceived effectiveness.
Fig. 5. Experiment \#1: pick and place. Overall mean and $95 \%$ confidence interval of (a) completion time and (b) motor load; (c) means of motor load split by object; (d) perceived effectiveness of the feedback conditions (the rating given by the subject could be in the range $1-10$, the higher values, the better performance).

Sphericity was assumed for the other factor, as it has only two levels. The two-way repeated-measure ANOVA did not reveal a statistically significant two-way interaction between feedback conditions and objects $\left(F_{4,60}=1.914, p=0.113\right)$. The main effect showed a statistically significant difference in motor load between conditions $\left(F_{1,15}=25.326, p<\right.$ $0.001)$ and objects $\left(F_{4,60}=93.587, p<0.001\right)$. According to the pairwise comparison, all objects lead to significantly different loads except dice vs. glass. Moreover, we ran a Pearson product-moment correlation test to study the relationship between the motor load exerted to lift each object and its weight. Regardless of the feedback condition considered, we found a strong positive correlation between these two variables: $r(3)=0.969, p=0.007$ and $r(3)=$ $0.964, p=0.008$ for the conditions $\mathrm{S}$ and $\mathrm{SH}$, respectively.

In addition to the quantitative evaluation reported above, we also measured users' experience. At the end of the experiment, subjects were asked to rate, on a slider going from 0 to 10 , the effectiveness of each feedback condition in completing the given task. Figure $5 \mathrm{~d}$ shows the perceived effectiveness for the three feedback conditions. A Friedman test showed a statistically significant difference between the means of the three feedback conditions $\left(\chi^{2}(2)=27.763, p<\right.$ 0.001). Post hoc analysis with Bonferroni adjustments revealed a statistically significant difference between $\mathrm{N}$ vs. $\mathrm{S}$ $(p=0.008)$ and $\mathrm{N}$ vs. SH $(p<0.001)$.

\section{B. Experiment \#2: ADL tasks with post-stroke patients}

Two chronic stroke patients (both males, age 35 and 56) took part to our experimental evaluation. The two subjects were required to wear the robotic finger on their paretic hand

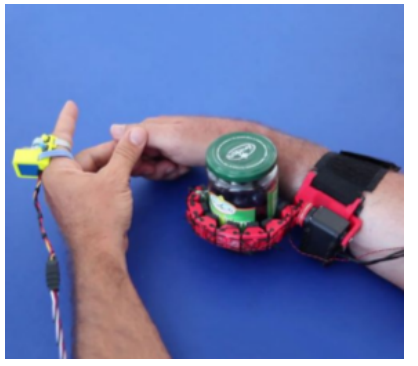

(a) Opening a jar of jam.

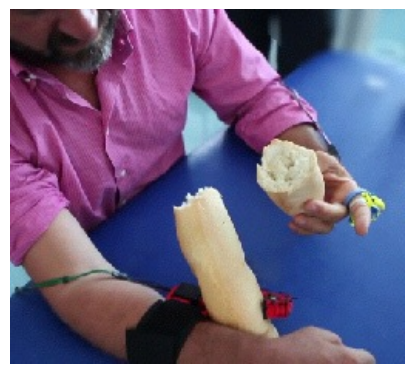

(b) Breaking a piece of bread.
Fig. 6. One chronic stroke patient performing ADL bi-manual tasks.

and the hRing on the healthy hand index finger (see Fig. 6). The proposed compensatory tool can be used by patients showing a residual mobility of the arm. For being included in this experimental evaluation, patients had to score $\leq 2$ when their motor functions were tested with the National Institute of Health Stroke Scale (NIHSS), item 5, "paretic arm". Moreover, the patients had to show the following characteristics: normal consciousness (NIHSS, item 1a, 1b, $1 \mathrm{c}=0$ ), absence of conjugate eyes deviation (NIHSS, item $2=0$ ), absence of complete hemianopia (NIHSS, item $3 \leq 1$ ), absence of ataxia (NIHSS, item $7=0$ ), absence of completely sensory loss (NIHSS, item $8 \leq 1$ ), absence of aphasia (NIHSS, item $9=0$ ), absence of profound extinction and inattention (NIHSS, item $11 \leq 1$ ).

The patients used the soft finger for bimanual tasks typical of Activities of Daily Living (ADL), such as opening a jar of jam, breaking a piece of bread, and opening a can of beans. In all the tasks, the patients used the robotic finger and the paretic limb to hold the object, while the healthy hand was used to perform the manipulation and to interact with the hRing. For example, the patient can hold the bread with the help of supernumerary robotic finger and non-functional arm, while using the healthy hand to break it (see Fig. 6b).

As described in Sec. IV, the hRing interface was used to both control the flexion/extension motion of the robotic finger and to provide the patient with haptic feedback about the forces exerted by the robotic finger on the environment and the status of the grasping procedure.

Both patients found the soft robotic finger useful in the considered ADL tasks. Moreover, they found the hRing intuitive to use and unobtrusive. Finally, they reported haptic feedback to be a valuable information to estimate the quality of the grasp. Both patients would like to be able to use it at home.

\section{Discussion, CONCLUSION, AND FUTURE WORK}

This paper introduced the design of a novel soft supernumerary robotic finger integrated with a wearable skin stretch cutaneous interface, called hRing. The supernumerary finger aims at restoring the grasping capabilities of post-stroke patients with a paretic hand. The hRing enables patients to easily control the motion of the robotic finger while providing them with haptic feedback about the status of the grasping action. 
To demonstrate the effectiveness of our system, we carried out two experiments, enrolling sixteen healthy subjects and two post-stroke patients in pick-and-place and ADL tasks, respectively. Healthy subjects were asked to mimic a paresis on their right hand by closing it in a fist. Results with the healthy subjects showed that the supernumerary finger can significantly help to improve the grasping capabilities of paretic hands. Indeed, no subject was able to complete the pick-and-place task without using the supernumerary finger. Moreover, results also show that providing cutaneous feedback through the hRing significantly improve the performance of the considered pick-and-place task in terms of force applied on the environment and perceived effectiveness. It is worth to point out that the heavier the object, the larger the improvement of performance when using the haptic feedback. Finally, the two chronic stroke patients found the system very useful for ADL tasks, the hRing easy to use, and the haptic feedback very informative. However, it is also important to notice that the benefits of using the proposed system are not always so evident. In fact, in our task, the only objects that all users were not able to grasp were the small cubes.

In the future, we are planning to add an ATI 6-DoF force/torque sensor on the supernumerary finger, in order to be able to accurately measure the forces and torques exerted by the finger on the environment. Moreover, using a 6-DoF sensor will enable us to fully exploit the capabilities of the hRing of providing both normal and skin stretch stimuli at the fingertip. In fact, in this work, since we only had the torque information coming from the load of the servomotor, we provided solely stimuli normal to the finger pad. We are also going to investigate how providing vibrotactile stimuli to other parts of the body (e.g., the wrist, the forearm) affects the performance of the considered tasks. Finally, we will extensively test our device in more challenging real-life scenarios, such as the Frenchay Arm Test.

\section{REFERENCES}

[1] A. S. Go, D. Mozaffarian, V. L. Roger, E. J. Benjamin, J. D. Berry, M. J. Blaha, S. Dai, E. S. Ford, C. S. Fox, S. Franco, et al., "Heart disease and stroke statistics-2014 update: a report from the american heart association," Circulation, vol. 129, no. 3, p. e28, 2014.

[2] H. Nakayama, H. S. Jorgensen, H. O. Raaschou, and T. S. Olsen, "Compensation in recovery of upper extremity function after stroke: the copenhagen stroke study," Archives of physical medicine and rehabilitation, vol. 75, no. 8, pp. 852-857, 1994.

[3] I. Faria-Fortini, S. M. Michaelsen, J. G. Cassiano, and L. F. TeixeiraSalmela, "Upper extremity function in stroke subjects: relationships between the intl. classification of functioning, disability, and health domains," Journal of Hand Therapy, vol. 24, no. 3, pp. 257-265, 2011.

[4] S. Balasubramanian, J. Klein, and E. Burdet, "Robot-assisted rehabilitation of hand function," Current opinion in neurology, vol. 23, no. 6, pp. 661-670, 2010.

[5] S. Masiero, A. Celia, G. Rosati, and M. Armani, "Robotic-assisted rehabilitation of the upper limb after acute stroke," Archives of physical medicine and rehabilitation, vol. 88, no. 2, pp. 142-149, 2007.

[6] A. Chiri, N. Vitiello, F. Giovacchini, S. Roccella, F. Vecchi, and M. C. Carrozza, "Mechatronic design and characterization of the index finger module of a hand exoskeleton for post-stroke rehabilitation," IEEE/ASME Trans. Mechatronics, vol. 17, no. 5, pp. 884-894, 2012.

[7] P. S. Lum, S. B. Godfrey, E. B. Brokaw, R. J. Holley, and D. Nichols, "Robotic approaches for rehabilitation of hand function after stroke,"
American Journal of Physical Medicine \& Rehabilitation, vol. 91, no. 11, pp. S242-S254, 2012.

[8] G. Kwakkel, B. J. Kollen, J. van der Grond, and A. J. Prevo, "Probability of regaining dexterity in the flaccid upper limb impact of severity of paresis and time since onset in acute stroke," Stroke, vol. 34, no. 9, pp. 2181-2186, 2003.

[9] D. Prattichizzo, M. Malvezzi, I. Hussain, and G. Salvietti, "The sixthfinger: a modular extra-finger to enhance human hand capabilities," in Proc. IEEE Int. Symp. in Robot and Human Interactive Communication, pp. 993-998, 2014.

[10] I. Hussain, G. Salvietti, L. Meli, C. Pacchierotti, D. Cioncoloni, S. Rossi, and D. Prattichizzo, "Using the robotic sixth finger and vibrotactile feedback for grasp compensation in chronic stroke patients," in Proc. IEEE/RAS-EMBS Intl. Conf. on Rehabilitation Robotics, pp. 67$72,2015$.

[11] I. Hussain, G. Spagnoletti, G. Salvietti, and D. Prattichizzo, "An emg interface for the control of motion and compliance of a supernumerary robotic finger," Frontiers in Neurorobotics, vol. 10, p. 18, 2016.

[12] I. Hussain, G. Salvietti, G. Spagnoletti, D. Cioncoloni, S. Rossi, and D. Prattichizzo, "A soft robotic extra-finger and arm support to recover grasp capabilities in chronic stroke patients," in Wearable Robotics: Challenges and Trends, Springer Intl. Publishing, 2016.

[13] G. Salvietti, I. Hussain, D. Cioncoloni, S. Taddei, S. Rossi, and D. Prattichizzo, "Compensating hand function in chronic stroke patients through the robotic sixth finger," IEEE Trans. Neural Systems and Rehabilitation Engineering, vol. 25, no. 2, pp. 142-150, 2017.

[14] I. Hussain, L. Meli, C. Pacchierotti, G. Salvietti, and D. Prattichizzo, "Vibrotactile haptic fedback for intuitive control of robotic extra fingers," in Proc. IEEE World Haptics Conf., pp. 394-399, 2015.

[15] I. Hussain, G. Spagnoletti, C. Pacchierotti, and D. Prattichizzo, "A wearable haptic ring for the control of extra robotic fingers," in Proc. Asia Haptics, (Chiba, Japan), 2016.

[16] L. Meli, C. Pacchierotti, and D. Prattichizzo, "Sensory subtraction in robot-assisted surgery: fingertip skin deformation feedback to ensure safety and improve transparency in bimanual haptic interaction," IEEE Trans. Biomedical Engineering, vol. 61, no. 4, pp. 1318-1327, 2014.

[17] C. Pacchierotti, D. Prattichizzo, and K. J. Kuchenbecker, "Cutaneous feedback of fingertip deformation and vibration for palpation in robotic surgery," IEEE Trans. Biomedical Engineering, vol. 63, no. 2, pp. 278287, 2016.

[18] C. Pacchierotti, Cutaneous haptic feedback in robotic teleoperation. Springer Series on Touch and Haptic Systems, Springer International Publishing, 2015.

[19] S. M. Son, Y. H. Kwon, N. K. Lee, S. H. Nam, and K. Kim, "Deficits of movement accuracy and proprioceptive sense in the ipsi-lesional upper limb of patients with hemiparetic stroke," Journal of physical therapy science, vol. 25, no. 5, p. 567, 2013.

[20] I. Hussain, G. Salvietti, M. Malvezzi, and D. Prattichizzo, "Design guidelines for a wearable robotic extra-finger," in Research and Technologies for Society and Industry Leveraging a better tomorrow (RTSI), 2015 IEEE 1st Intl. Forum on, pp. 54-60, Sept 2015.

[21] I. Hussain, G. Salvietti, G. Spagnoletti, and D. Prattichizzo, "The soft-sixthfinger: a wearable emg controlled robotic extra-finger for grasp compensation in chronic stroke patients," IEEE Robotics and Automation Letters, vol. 1, no. 2, pp. 1000-1006, 2016.

[22] A. M. Dollar and R. D. Howe, "Joint coupling design of underactuated hands for unstructured environments," The Intl. Journal of Robotics Research, vol. 30, no. 9, pp. 1157-1169, 2011.

[23] C. Eppner and O. Brock, "Grasping unknown objects by exploiting shape adaptability and environmental constraints," in Proc. IEEE/RSJ Intl. Conf. Intelligent Robots and Systems, pp. 4000-4006, 2013.

[24] C. Pacchierotti, S. Sinclair, M. Solazzi, A. Frisoli, V. Hayward, and D. Prattichizzo, "Wearable haptic systems for the fingertip and the hand: taxonomy, review, and perspectives," IEEE Trans. Haptics. In Press, 2017.

[25] K. Minamizawa, S. Fukamachi, H. Kajimoto, N. Kawakami, and S. Tachi, "Gravity grabber: wearable haptic display to present virtual mass sensation," in Proc. ACM Special Interest Group on Computer Graphics and Interactive Techniques Conf., p. 8, 2007.

[26] D. Prattichizzo, C. Pacchierotti, and G. Rosati, "Cutaneous force feedback as a sensory subtraction technique in haptics," IEEE Trans. Haptics, vol. 5, no. 4, pp. 289-300, 2012.

[27] K. H. Park, B. H. Kim, and S. Hirai, "Development of a softfingertip and its modeling based on force distribution," in Proc. IEEE International Conf. on Robotics and Automation, vol. 3, pp. 31693174, 2003. 